\title{
Identification of Mycobacterium tuberculosis clinical isolates in Bangladesh by a species distinguishable multiplex PCR
}

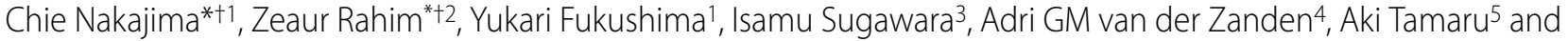 \\ Yasuhiko Suzuki*1
}

\begin{abstract}
Background: Species identification of isolates belonging to the Mycobacterium tuberculosis complex (MTC) seems to be important for the appropriate treatment of patients, since $M$. bovis is naturally resistant to a first line anti-tuberculosis (TB) drug, pyrazinamide, while most of the other MTC members are susceptible to this antimicrobial agent. A simple and low-cost differentiation method was needed in higher TB burden countries, such as Bangladesh, where the prevalence of $M$. bovis among people or cattle has not been investigated.

Methods: Genetic regions cfp32, RD9 and RD12 were chosen as targets for a species distinguishable multiplex PCR and the system was evaluated with twenty reference strains of mycobacterial species including non-tubercular mycobacteria (NTM). A total of 350 clinical MTC isolates obtained in Bangladesh were then analyzed with this multiplex PCR.

Results: All of the MTC reference strains gave expected banding patterns and no non-specific amplifications were observed in the NTM strains. Out of 350 clinical isolates examined by this method, 347 (99.1\%) were positive for all of the cfp32, RD9 and RD12 and determined as M. tuberculosis. Two isolates lacked cfp32 PCR product and one lacked RD12, however, those three samples were further examined and identified as M. tuberculosis by the sequence analyses of hsp65 and gyrB.

Conclusions: The MTC-discrimination multiplex PCR (MTCD-MPCR) developed in this study showed high specificity and was thought to be very useful as a routine test because of its simplicity. In the current survey, all the 350 MTC isolates obtained from Bangladesh TB patients were determined as M. tuberculosis and no other MTC were detected. This result suggested the general TB treatment regimen including pyrazinamide to be the first choice in Bangladesh.
\end{abstract}

\section{Background}

Mycobacterium tuberculosis complex (MTC), including M. tuberculosis, M. bovis, M. africanum, M. microti, $M$. pinnipedii, M. caprae, "M. canettii" and other closely related strains, is a group of causative agents for human and animal tuberculosis (TB) [1,2]. Although the mycobacterial species in MTC are highly similar to each other in DNA level, MTC members differ widely in terms of host tropism, phenotype and pathogenicity [1,3-5]. No further differentiation is usually performed with isolates

\footnotetext{
* Correspondence: cnakajim@czc.hokudai.ac.jp

1 Department of Global epidemiology, Hokkaido University Research Center for Zoonosis Control, Kita20-Nishi10, Kita-ku, Sapporo 001-0020, Japan + Contributed equally

Full list of author information is available at the end of the article
}

determined as MTC, however, it seems to be important in some cases for the appropriate management of patients or for an epidemiological purpose. Especially, in the case of $M$. bovis infection, to identify the species in the early stage of diagnosis is essential to avoid inappropriate treatment, since $M$. bovis is naturally resistant to a major antiTB drug, pyrazinamide $[3,4,6]$, and the standard regimen including this drug has to be altered.

Several rapid identification methods using nucleic acid amplification techniques have been developed and used for the diagnosis of TB [7-9], however, they do not differentiate M. tuberculosis from other members of MTC. Recent comparative genomic analyses have provided valuable information on the region of difference (RD) in 
the chromosome of MTC to indicate that specific identification of MTC can be achieved by the detection of these regions $[1-3,10]$. PCR-based methods targeting RDs can be easily performed in local clinical laboratories with low expense [3].

Bangladesh is one of the highest TB burden countries, where the estimated number of TB incidence in 2007 was 353,000 to be ranked sixth in the world in the WHO report [11]. In this country, a large number of cattle estimated 23 million heads are reared in households especially in rural areas [12]. Some surveys about drug resistant $M$. tuberculosis $[13,14]$ or $M$. tuberculosis epidemiology [15] have been performed, however, no survey about $M$. bovis prevalence among humans as well as cattle has been reported though people are living in a close relationship with cattle $[12,16]$.

In this study, we developed a simple multiplex-PCR system, named MTC-discrimination multiplex PCR (MTCD-MPCR), to distinguish $M$. tuberculosis from other MTC species using RDs, and applied it for clinical isolates derived from TB patients in Bangladesh.

\section{Methods}

\section{Bacterial strains and sample collection}

For the evaluation of the method, following twenty reference strains, four MTC strains and sixteen nontuberculous mycobacteria (NTM) strains, were obtained from the Research Institute of Tuberculosis, Japan Anti-Tuberculosis Association (Tokyo, Japan) and used: M. tuberculosis H37Rv, M. africanum KK13-02, M. microti ATCC19422, M. bovis BCG Tokyo, M. avium JATA51-01, $M$. intracellulare JATA52-01, M. kansasii KK21-01, $M$. xenopi KK42-02, M. fortuitum JATA61-01, M. lentiflavum JATA9N-01, $M$. simiae KK23-01, M. gordonae JATA33-01, $M$. marinum JATA22-01, $M$. asiaticum KK24-01, M. scrofulaceum JATA31-01, M. szulgai JATA32-01, M. nonchromogenicum JATA45-01, M. malmoense JATA47-01, M. chelonae JATA62-01 and $M$. abscessus JATA63-01.

Clinical samples were collected in hospitals in Dhaka, located in an urban area, and Matlab and Sylhet, located in rural areas. A total of 350 isolates, 300 from Dhaka, 41 from Matlab and 9 from Sylhet, were examined (Additional file 1). Among them, 327 isolates were derived from sputa, 22 were from lymph nodes and remaining 1 was from a surgical injury.

\section{Cultivation and biochemical characterization of isolates and DNA extraction}

Sputa and other samples were collected from TB suspected patients and decontaminated following the Petropff's method [17]. After a centrifugation at $500 \mathrm{rpm}$ at $4^{\circ} \mathrm{C}$, the supernatant was discarded and one loop-full ( 5 $\mathrm{mm}$ diameter of the loop) decontaminated pellet was inoculated onto 2 Lowenstein-Jensen (L-J) slants each. Inoculated L-J slants were incubated at $37^{\circ} \mathrm{C}$. Each L-J slant was examined once a week for contamination as well as for growth until 8 weeks. Typical mycobacteriumlike colonies were tested for sensitivity to p-nitrobenzoic (PNB) acid. PNB sensitive strains were considered to be M. tuberculosis complex. DNA was extracted from those colonies by heating at $95^{\circ} \mathrm{C}$ for 5 min followed by chloroform extraction and ethanol precipitation [18].

\section{MTC-discrimination multiplex PCR (MTCD-MPCR)}

Primer pairs for $c f p 32$ (Rv0577), RD9 (Rv2073c) and RD12 (Rv3120) designed by Huard et al [3] were slightly modified and used as a primer mixture for three simultaneous PCRs in one tube (Table 1). The reaction mixture contained $1 \mathrm{mM}$ dNTPs (0.25 mM each), $0.5 \mathrm{M}$ betaine, $750 \mathrm{nM}$ each of $c f p 32$ primers (Rv0577F and Rv0577R), $250 \mathrm{nM}$ each of RD primers (Rv2073cF, Rv2073cR, Rv3120F and Rv3120R (390-369)), $1 \mu \mathrm{L}$ of DNA sample and $1 \mathrm{U}$ of GoTaq DNA Polymerase (Promega Corp., WI, U.S.A.) in $20 \mu \mathrm{L}$ of Green GoTaq Reaction Buffer. PCR reaction was initiated by denaturation for $1 \mathrm{~min}$ at $96^{\circ} \mathrm{C}$, followed by 35 cycles of $10 \mathrm{~s}$ at $96^{\circ} \mathrm{C}, 20 \mathrm{~s}$ at $60^{\circ} \mathrm{C}$ and 1 min at $72^{\circ} \mathrm{C}$ with final extension for $5 \mathrm{~min}$ at $72^{\circ} \mathrm{C}$ in a thermalcycler (iCycler, Bio Rad Laboratories Inc., CA, U.S.A.). Reaction mixtures with M. tuberculosis DNA and without template DNA were also run simultaneously with samples every time as a positive control and a negative control to evaluate the MTCD-MPCR system. The products were electrophoresed in $2.0 \%$ agarose gel in TAE buffer, and stained with ethidium bromide.

The sensitivity of the method was determined using serially diluted purified genomic-DNA solutions, ten-fold dilution from $5 \mathrm{ng} / \mu \mathrm{L}$ to $50 \mathrm{fg} / \mu \mathrm{L}$, extracted from $M$. tuberculosis $\mathrm{H} 37 \mathrm{Rv}$ and M. bovis BCG Tokyo. For the specificity study, the concentration of the DNA solution from each reference strain was adjusted to $5 \mathrm{ng} / \mu \mathrm{L}$ and used.

A detection study from sputum samples was performed with $M$. tuberculosis H37Rv spiked sputa. Serially diluted bacteria were spiked into healthy volunteer's sputum samples to final concentrations ranging from $1.5 \times 10 / \mathrm{mL}$ to $1.5 \times 10^{6} / \mathrm{mL}$. The sputum was processed by a conventional method and DNA was extracted by bead-beating. Briefly, twice the volume of $\mathrm{N}$-acetyl-L-cysteine and $\mathrm{NaOH}$ (NALC-NaOH) was added to the sputum, mixed well and incubated for 15 minutes. The sample was diluted to five times its original volume with PBS and centrifuged for $20 \mathrm{~min}$ at $3000 \mathrm{rpm}$. The sediment was dissolved in $0.75 \mathrm{~mL}$ of Tris-EDTA buffer, added $0.5 \mathrm{~g}$ of glass beads (0.15 - $0.25 \mathrm{~mm}$, Fuji Chemical Industry Co., Ltd., Japan) and $0.5 \mathrm{~mL}$ of chloroform and then shaken with a bead-beater (FastPrep FP100A, MP Biomedicals, U.S.A.) for $5 \mathrm{~min}$ at $5500 \mathrm{rpm}$. Tubes were centrifuged at 
$5000 \mathrm{rpm}$ for $5 \mathrm{~min}$ and $0.5 \mathrm{~mL}$ of PCI (phenol-chloroform-isoamyl alcohol, 25:24:1) was added to the supernatant, mixed and centrifuged. DNA was extracted from the PCI treated sample by isopropyl alcohol precipitation and the precipitant was dissolved in $10 \mu \mathrm{L}$ of Tris-EDTA buffer. Final solutions were subjected to the MTCD-MPCR.

\section{Other PCRs and Sequence analyses}

Additional PCRs and sequencings were performed with primers listed in Table 1. Reaction solution components in those PCRs were as follows: $1 \mathrm{mM}$ dNTPs $(0.25 \mathrm{mM}$ each), $0.5 \mathrm{M}$ betaine, $500 \mathrm{nM}$ each of forward and reverse primers, $1 \mu \mathrm{L}$ of DNA sample and $1 \mathrm{U}$ of GoTaq DNA Polymerase (Promega Corp.) in $20 \mu \mathrm{L}$ of Green GoTaq Reaction Buffer. PCR reaction other than rrs was initiated by denaturation for $1 \mathrm{~min}$ at $96^{\circ} \mathrm{C}$, followed by 30 cycles of $10 \mathrm{~s}$ at $96^{\circ} \mathrm{C}, 10 \mathrm{~s}$ at $55^{\circ} \mathrm{C}$ and $30 \mathrm{sec}$ at $72^{\circ} \mathrm{C}$ with final extension for $5 \mathrm{~min}$ at $72^{\circ} \mathrm{C}$ in a thermalcycler (iCycler, Bio Rad Laboratories Inc.). In rrs PCR, the period of extension at $72^{\circ} \mathrm{C}$ in the cycle was $90 \mathrm{sec}$, whereas other conditions were same with other PCR procedure. The products were electrophoresed in 1.5\% agarose gel and stained with ethidium bromide. Sequencing of PCR product was performed according to manufacturer's protocol with ABI PRISM 3130 $x$ l Genetic Analyzer (Life Technologies Corp., CA, U.S.A.) using BigDye Terminator v3.1 Cycle Sequencing Kit (Life Technologies Corp.). rpoB and rrs sequences read by each forward primer (Table $1 . b$ ) were compared with the sequence of $M$. tuberculosis $\mathrm{H} 37 \mathrm{Rv}$ and the samples met following criteria were identified as MTC: $r p o B$, more than $98 \%$ match in minimum 150-base length; rrs, 100\% match in minimum 300 bases. About the samples determined as non-MTC by these criteria, another PCR and sequencing were done with more

Table 1: Used primers for the MTCD-MPCR and additional PCRs and sequencings.

\begin{tabular}{|c|c|c|c|c|c|}
\hline Target locus & Primer name & Primer sequence & Locationa & Size (bp) & Ref. No. \\
\hline \multicolumn{6}{|l|}{ MTCD-MPCR } \\
\hline \multirow[t]{2}{*}{ cfp32 } & Rv0577F & 5' ATGCCCAAGAGAAGCGAATACAGGCAA & 671166-192 & 786 & [3] \\
\hline & Rv0577R & 5' CTATTGCTGCGGTGCGGGCTTCAA & $671951-928$ & & \\
\hline \multirow[t]{2}{*}{ RD9 } & Rv2073cF & 5' TCGCCGCTGCCAGATGAGTC & $2330579-598$ & 600 & [3] \\
\hline & Rv2073cR & 5' TाTGGGAGCCGCCGGTGGTGATGA & $2331173-150$ & & \\
\hline \multirow[t]{2}{*}{ RD12 } & Rv3120F & 5' GTCGGCGATAGACCATGAGTCCGTCTCCAT & $3485558-587$ & 404 & [3] \\
\hline & Rv3120R (390-369) & 5' GCGAAAAGTGGGCGGATGCCAG & $3485961-940$ & & \\
\hline \multicolumn{6}{|c|}{ Additional PCRs or sequencings } \\
\hline \multirow[t]{2}{*}{ cfp32 } & 3'cfp32F & 5' CGAATCATTGGCACGTCTACTITG & $671770-793$ & 372 & [2] \\
\hline & 3'cfp32R & 5' GTGGCACCGGCGGCACCGCACACCT & $672141-117$ & & \\
\hline \multirow[t]{2}{*}{ RD12 } & Rv3120-F (90-110) & 5' GGTATTTGCGCCCATATCCTG & $3485661-681$ & 411 & this study \\
\hline & Rv3120-R (500-481) & 5' CCTGGCTTCAAGCACCATTC & $3486071-052$ & & \\
\hline \multirow[t]{2}{*}{$r p o B$} & $r p o B-F^{b}$ & 5' CAGGACGTGGAGGCGATCAC & $761007-026$ & 250 & {$[18]$} \\
\hline & $r p o B-\mathrm{R}$ & 5' CAGGGGTTTCGATCGGGCAC & $761256-237$ & & \\
\hline \multirow[t]{2}{*}{$r p o B^{c}$} & $r p o B-\mathrm{S}-\mathrm{F} b$ & 5' GCGTACGGTCGGCGAGCTGATCC & $760922-944$ & 418 & this study \\
\hline & rpoB-S-R & 5' GCGGTACGGCGTTTCGATGAACC & $761339-317$ & & \\
\hline \multirow[t]{2}{*}{ rrs } & Bact-rrs- $\mathrm{F}^{b}$ & 5' AGAGTTTGATCCTGGCTCAG & $1471856-875$ & 1496 & this study \\
\hline & Bact-rrs-R & 5' TACGGCTACCTTGTTACGAC & $1473351-332$ & & \\
\hline \multirow[t]{2}{*}{$r r s^{c}$} & $r r s-\mathrm{S}-\mathrm{F}^{b}$ & 5' ATACCTTTGGCTCCCTITTCC & $1471809-829$ & 1607 & this study \\
\hline & $r r s-S-R$ & 5' CCCACCAGTTGGGGCGTTITC & 1473415-395 & & \\
\hline \multirow[t]{2}{*}{ hsp65 } & $h s p 65 \mathrm{~F}^{b}$ & 5' ACCAACGATGGTGTGTCCAT & $528752-771$ & 441 & {$[2]$} \\
\hline & hsp65R & 5' CTTGTCGAACCGCATACCCT & $529192-173$ & & \\
\hline \multirow[t]{2}{*}{ gyrB } & $g y r B F b$ & 5' ACATCAACCGCACCAAGAACGC & $6027-048$ & 483 & this study \\
\hline & gyrBR & 5' GTGCCTTACGTGCCGCGATACG & $6509-488$ & & \\
\hline
\end{tabular}

a Location on the M. tuberculosis H37Rv genome (accession no. NC_000962.2).

b These primers were used for PCR and sequencing.

c Those primer pairs were used for the samples that showed non-MTC gene sequences. 
MTC specific primers (Table 1.c) with the same criteria. The samples identified as MTC with those specific primers were determined as mixed-culture samples. Species identifications by $h s p 65$ or $\operatorname{gyr} B$ sequences were done according to previous publications $[2,19,20]$.

\section{Ethical Clearance}

The original research project was approved by the Research Review Committee and Ethical Review Committee of the International Centre for Diaddroeal Disease Research, Bangladesh (ICDDR, B). Signed informed consent was obtained from each patient and volunteer recruited for the study.

\section{Results and discussion MTCD-MPCR}

Three genetic regions were selected as the targets for the multiplex PCR: $c f p 32, \mathrm{RD} 9$ and RD12. $c f p 32$ is an MTCrestricted gene and used to confirm isolates to belong to MTC $[3,21]$. RD9 is the region that can be found in only M. tuberculosis and "M. canettii", and RD12 is found in all MTC members except M. bovis, M. caprae and "M. canet$t i i^{\prime \prime}[2,3]$. By a trial with several patterns of primer concentrations, the best combination was determined to be $750 \mathrm{nM}$ each of $c f p 32$ primers and $250 \mathrm{nM}$ each of RD9 and RD12 primers in the multiplex PCR. With this PCR, an isolate possessing all the three regions can be identified as $M$. tuberculosis whereas a strain showing only one amplified band, $c f p 32$, will be classified as M. bovis or M. caprae (Figure 1, Table 2). Other banding patterns are interpreted as described in Table 2. "M. canettii" is another MTC that clinical isolates reported so far were naturally pyrazinamide resistant $[6,19]$. Thus, this PCR system was thought to be useful for the discrimination of MTC species, especially for the screening of naturally pyrazinamide-resistant species with only one PCR reaction per sample.

The sensitivities of the method were determined as 500 fg genomic DNA for M. tuberculosis (H37Rv) and $50 \mathrm{fg}$ for M. bovis (BCG Tokyo), which were assumed to be equivalent to 100 bacilli and 10 bacilli, respectively (data not shown). The specificity of the MTCD-MPCR was confirmed with DNA templates extracted from mycobacterial reference strains. Typical gel electrophoresis results of the MTC and NTM strains are shown in Figure 1. All of the PCR products from the MTC strains gave expected banding patterns in correct sizes (Table 1). On the contrary, no bands were obtained from the NTM samples. These results demonstrated the specificity and applicability of the MTCD-MPCR for the differentiation of M. tuberculosis in clinical isolates.

In the study using bacterium-spiked sputa, the detection limit of M. tuberculosis was $1.5 \times 10^{4}$ cells in $1 \mathrm{~mL}$ of sputum (data not shown). This bacterial concentration is similar or a little higher than the detection limit of the bacteria by the Zeal-Nelsen staining. This means species discrimination by the MTCD-MPCR is applicable for clinical samples if the sputum is diagnosed as smear positive. The discrepancy of the detection limit between purified DNA and bacterium-spiked sputum seems to depend on the extraction or purification procedure, which is to be improved.

\section{Discrimination of MTC in Bangladesh clinical isolates}

A total of 350 clinical isolates obtained in Bangladesh were analyzed to see the prevalence of MTC species other than $M$. tuberculosis. All samples were subjected to $r p o B$ or rrs sequencing to confirm as MTC [22]. By this sequence analysis, 18 samples were revealed as mixtures of MTC and other bacteria, mainly mycobacterial species. Those mixed samples were also subjected to the MTCD-MPCR study.

Out of the 350 isolates, 347 (99.1\%) showed the typical banding pattern of $M$. tuberculosis in the electrophoresis by the MTCD-MPCR (Table 2). Among three remaining isolates, one (0.3\%, isolate ATP138) lacked RD12 band showing "M. canettii" pattern and two $(0.6 \%$, isolate M2000 and S2247) did not have cfp32 band, presented as irregular in Table 2. Those samples were further analyzed to detect the target region, $c f p 32$ or RD12, with other sets of primers (Table 1) [2]. With this trial, sample S2247 gave an expected sized $c f p 32$ band, suggesting a partial deletion or a mutation in the primer-binding site. Other two samples, ATP138 and M2000, still failed to amplify the expected DNA fragment indicating that larger deletion event had occurred in the target genomic area. ATP138, the "M. canettii" pattern sample, was subjected to hsp 65 sequencing and confirmed not to be "M. canet$t i i "[2,19]$. These three isolates were further confirmed to be $M$. tuberculosis by the sequencing of $g y r B$ [20]. No isolates out of the 350 lacked RD9 band, concordant with the observations by former researchers $[2,10,23]$, possibly indicating the high stability of this region in M. tuberculosis. The lack of $c f p 32$ in an MTC isolate has been reported by Huard et al [2] with a similar incidence $(1 / 125,0.8 \%)$ to the current study. Some of the mixed-culture samples showed correct but faint banding patterns, suggesting an inhibitory effect of contaminated DNA. The result indicated that despite its decreased sensitivity, the MTCDMPCR was able to detect MTC from mixed-culture samples, which sometimes are observed in primary cultures [8].

All of the 350 MTC isolates obtained from clinical specimens in Bangladesh were M. tuberculosis and no other MTC species were detected. This information is very helpful for the management of patients to determine treatment regimens. Patients suffered from MTC in surveyed area in Bangladesh can possibly be subjected to the 
standard regimen including pyrazinamide. Although $M$. bovis was not detected from human in current study, there are no precise data about $M$. bovis prevalence among cattle in this country, continuous surveys seem to be needed especially in rural areas where people and cattle inhabit more closely [12]. Since pyrazinamide is suggested to have more adverse side effects than other firstline anti-TB drugs [24], useless administration to patients should be avoided.

The MTCD-MPCR developed in this study is considered to be very useful for the differentiation of MTC because of its simplicity and specificity. A large number of samples can be analyzed by this method in a short period of time. Some other MTC discrimination methods using RDs have been published and showed higher differentiation capability that could distinguish almost all members of MTC. However, the procedures were more time consuming (e.g., multiple PCR reactions were needed) [2,3], result interpretations seemed to be complicated (e.g., sizes of amplified bands should be estimated) $[10,23,25]$ or an expensive equipment should be needed [26]. The necessity of detailed MTC discrimination seems to be low since the majority of human tuberculosis causing agents are $M$. tuberculosis, and in some global areas, $M$. bovis partially contributes to the prevalence $[4,23]$. The vaccine strain bacillus Calmette-Guerin (BCG), an attenuated $M$. bovis, can be a cause of disseminated mycobacterial infection in immunocompromised individuals, however, the patients can be treated by the same regimen as $M$. bovis without pyrazinamide [27], and if necessary, an additional PCR for the detection of RD1 can distinguish BCG from clinical M. bovis strains [3]. Possibility of the detection of other MTC species is almost

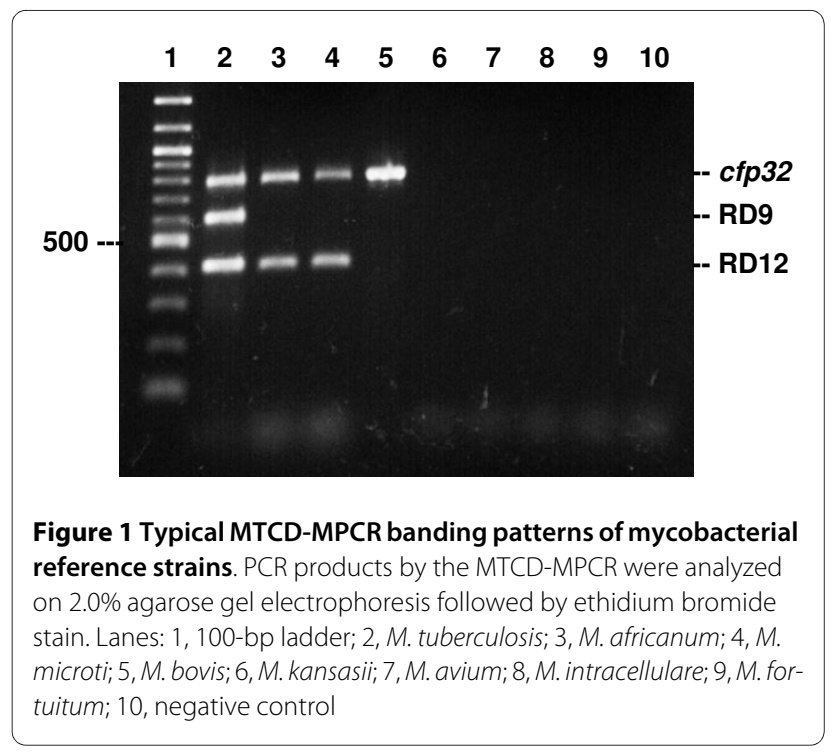

negligible in routine laboratory diagnoses, except for the cases in some African countries where $M$. africanum can be found in a higher ratio [2]. Samples exhibiting rare or irregular banding patterns by the MTCD-MPCR can be examined afterward using other precise methods, e.g., targeting other RDs, gene sequencing or spoligotyping $[2,19,20,28]$. Thus, the MTCD-MPCR, a simple MTC discrimination method developed and evaluated in this study seemed to be very useful as a screening tool for clinical isolates to distinguish $M$. tuberculosis from $M$. bovis for the prompt decision of treatment regimen.

\section{Conclusions}

In the current study, the MTC-discrimination multiplex PCR (MTCD-MPCR) was developed and applied for a

Table 2: Results of the MTCD-MPCR with Bangladesh clinical isolates.

\begin{tabular}{|c|c|c|c|c|c|}
\hline \multirow{2}{*}{$\begin{array}{l}\text { Species } \\
\text { Interpretation }\end{array}$} & \multicolumn{3}{|c|}{ Banding pattern $a$} & \multirow{2}{*}{$\begin{array}{c}\text { Number of } \\
\text { isolates }\end{array}$} & \multirow[t]{2}{*}{$\%$} \\
\hline & cfp32 & RD9 & RD12 & & \\
\hline M. tuberculosis & + & + & + & 347 & 99.1 \\
\hline M. bovis/M. caprae & + & - & - & 0 & 0 \\
\hline "M. canettii" & + & + & - & $1^{c}$ & 0.3 \\
\hline Other MTC ${ }^{b}$ & + & - & + & 0 & 0 \\
\hline Non MTC & - & - & - & 0 & 0 \\
\hline Irregular & - & + & + & $2 d$ & 0.6 \\
\hline Total & & & & 350 & 100 \\
\hline $\begin{array}{l}\text { a Banding patterns } \\
\text { negative. } \\
\text { b Other MTC include } \\
\text { belonging to MTC } \\
\text { c Confirmed to be } M \\
\text { d Confirmed to be } N\end{array}$ & $\begin{array}{l}\text { as ampl } \\
\text { g specie } \\
\text { osis by hs } \\
\text { osis by gy }\end{array}$ & $\begin{array}{l}\text { of the } \mathrm{N} \\
\text { 1. micro } \\
\text { quencin }\end{array}$ & der of $c f$ & $\begin{array}{l}\text { RD12: +, Amp } \\
\text { x bacillus. The }\end{array}$ & $\begin{array}{l}\text { tive; -, } \\
\text { ninor strains }\end{array}$ \\
\hline
\end{tabular}


study to see the prevalence of MTC species other than $M$. tuberculosis in clinical isolates in Bangladesh. The method showed high specificity and sensitivity, as $99.1 \%$ (347/350) of clinical M. tuberculosis isolates were identified by a typical banding pattern. It seemed to be very useful as a routine test method because of its simplicity. All the 350 MTC isolates derived from Bangladesh patients were M. tuberculosis and no other MTC was detected. The result suggested that a standard TB treatment regimen including pyrazinamide can be applied to the patients as the first choice in surveyed areas in Bangladesh.

\section{Additional material}

\section{Additional file 1 Used isolates in current study. Background informa-} tion of used isolates including sampling site and drug-susceptibility test results.

\section{Competing interests}

The authors declare that they have no competing interests.

\section{Authors' contributions}

$\mathrm{CN}, \mathrm{ZR}$ and $\mathrm{YS}$ were responsible for planning the study, analyzing the results and drafting the manuscript. YF carried out the molecular genetic studies. AT performed the detection study from sputum samples. ZR, IS, AGMZ and YS collected the study material and coordinated the study. All authors read and approved the manuscript.

\section{Acknowledgements}

This work was supported by the International Centre for Diarrhoeal Disease Research, Bangladesh, grants received from Gates-Government of the Peoples Republic of Bangladesh to ZR, Grants-in-Aid for Program of Founding Research Center for Emerging and Reemerging Infectious Diseases from the Ministry of Education, Culture, Sports, Science, and Technology, Japan (MEXT) to YS, and in part by the Global Center of Excellence (COE) Program, "Establishment of International Collaboration Centers for Zoonosis Control" from MEXT to YS and a grant from U.S.-Japan Cooperative Medical Science Programs to IS and YS.

\section{Author Details}

'Department of Global epidemiology, Hokkaido University Research Center for Zoonosis Control, Kita20-Nishi10, Kita-ku, Sapporo 001-0020, Japan, ${ }_{2}^{2}$ Tuberculosis laboratory, International Centre for Diarrhoeal Disease Research, Bangladesh (ICDDR, B), GPO Box 128, Dhaka 1000, Bangladesh, ${ }^{3}$ Research Institute of Tuberculosis, Japan Anti-Tuberculosis Association, 3-1-24 Matsuyama, Kiyose, Tokyo, Japan, ${ }^{4}$ Laboratory for Medical Microbiology and Public Health, P.O.Box 377, Burg. Edo Bergsmalaanl, 7512 AD Enschede, The Netherlands and ${ }^{5}$ Osaka Prefectural Institute of Public Health, 1-3-69, Nakamichi, Higashinari-ku, Osaka 537-0025, Japan

Received: 26 August 2009 Accepted: 15 May 2010

Published: 15 May 2010

\section{References}

1. Brosch R, Gordon SV, Marmiesse M, Brodin P, Buchrieser C, Eiglmeier K, Garnier T, Gutierrez C, Hewinson G, Kremer K, Parsons LM, Pym AS, Samper $S$, van Soolingen D, Cole ST: A new evolutionary scenario for the Mycobacterium tuberculosis complex. Proc Natl Acad Sci USA 2002, 99:3684-3689.

2. Huard RC, Fabre M, de Haas P, Lazzarini LC, van Soolingen D, Cousins D, Ho $J \mathrm{~L}$ : Novel genetic polymorphisms that further delineate the phylogeny of the Mycobacterium tuberculosis complex. J Bacteriology 2006, 188:4271-4287.

3. Huard RC, de Oliveira Lazzarini LC, Butler WR, van Soolingen D, Ho JL: PCRbased method to differentiate the subspecies of the Mycobacterium tuberculosis Complex on the basis of genomic deletions. J Clin Microbiol 2003, 41:1637-1650.

4. Smith NH, Gordon SV, de la Rua-Domenech R, Clifton-Hadley RS, Hewinson RG: Bottlenecks and broomsticks: the molecular evolution of Mycobacterium bovis. Nat Rev Microbiol 2006, 4:670-681.

5. Sreevatsan S, Pan X, Stockbauer KE, Connell ND, Kreiswirth BN, Whittam TS, Musser JM: Restricted structural gene polymorphism in the Mycobacterium tuberculosis complex indicates evolutionarily recent global dissemination. Proc Natl Acad Sci USA 1997, 94:9869-9874.

6. Somoskovi A, Dormandy J, Parsons LM, Kaswa M, Goh KS, Rastogi N, Salfinger M: Sequencing of the pncA gene in members of the Mycobacterium tuberculosis complex has important diagnostic applications: Identification of a species-specific pncA mutation in "Mycobacterium canettii" and the reliable and rapid predictor of pyrazinamide resistance. J Clin Microbio/ 2007, 45:595-599.

7. Jonas V, Alden MJ, Curry Jl, Kamisango K, Knott CA, Lankford R, Wolfe JM, Moore DF: Detection and identification of Mycobacterium tuberculosis directly from sputum sediments by amplification of rRNA. J Clin Microbiol 1993, 31:2410-2416.

8. Katila ML, Katila P. Erkinjuntti-Pekkanen R: Accelerated detection and identification of mycobacteria with MGIT 960 and COBAS AMPLICOR systems. J Clin Microbiol 2000, 38:960-964.

9. Pandey BD, Poudel A, Yoda T, Tamaru A, Oda N, Fukushima Y, Lekhak B, Risal B, Acharya B, Sapkota B, Nakajima C, Taniguchi T, Phetsuksiri B, Suzuki $Y$ : Development of an in-house loop-mediated isothermal amplification (LAMP) assay for detection of Mycobacterium tuberculosis and evaluation in sputum samples of Nepalese patients. J Med Microbiol 2008, 57:439-443.

10. Parsons LM, Brosch R, Cole ST, Somoskovi A, Loder A, Bretzel G, van Soolingen D, Hale YM, Salfinger M: Rapid and simple approach for identification of Mycobacterium tuberculosis complex isolates by PCRbased genomic deletion analysis. J Clin Microbiol 2002, 40:2339-2345

11. WHO: Epidemiology. WHO Report 2009 Global Tuberculosis Control 2009:6-33.

12. Haque A: Bangladesh: Social gains from dairy development. In Smallholder dairy development: Lessons learned in Asia Animal Production and Health Commission for Asia and the Pacific, Food and Agriculture Organization of the United Nations, Regional Office for Asia and the Pacific; 2009:8-21.

13. Storla DG, Rahim Z, Islam MA, Plettner S, Begum V, Myrvang B, Bjune G, Rønnild E, Dahle UR, Mannsåker T: Drug resistance of Mycobacterium tuberculosis in the Sunamganj District of Bangladesh. Scand J Infect Dis 2007, 39:142-145.

14. Zaman K, Rahim Z, Yunus M, Arifeen SE, Baqui AH, Sack DA, Hossain S, Banu S, Islam MA, Ahmed J, Breiman RE, Black RE: Drug resistance of Mycobacterium tuberculosis in selected urban and rural areas in Bangladesh. Scand J Infect Dis 2005, 37:21-26.

15. Rahim Z, Zaman K, Zanden AG van der, Möllers MJ, van Soolingen D, Raqib R, Zaman K, Begum V, Rigouts L, Portaels F, Rastogi N, Sola C: Assessment of population structure and major circulating phylogeographical clades of Mycobacterium tuberculosis complex in Bangladesh suggests a high prevalence of a specific subclade of ancient M. tuberculosis genotypes. J Clin Microbiol 2007, 45:3791-3794.

16. Rahim Z, Möllers M, te Koppele-Vije A, de Beer J, Zaman K, Matin MA Kamal M, Raquib R, van Soolingen D, Baqi MA, Heilmann FG, Zanden AG van der: Characterization of Mycobacterium africanum subtype I among cows in a dairy farm in Bangladesh using spoligotyping. Southeast Asian J Trop Med Public Health 2007, 38:706-713.

17. Petroff SA: A new and rapid method for isolation and culture of tubercle bacilli directly from the sputum and feces. J Exp Med 1915, 21:38-42

18. Suzuki Y, Katsukawa C, Inoue K, Yin Y, Tasaka H, Ueba N, Makino M: Mutations in rpoB gene of rifampicin resistant clinical isolates of Mycobacterium tuberculosis in Japan. Kansenshogaku Zasshi 1995, 69:413-419.

19. Goh KS, Legrand E, Sola C, Rastogi N: Rapid differentiation of "Mycobacterium canettii" from other Mycobacterium tuberculosis complex organisms by PCR-restriction analysis of the $h s p 65$ gene. $J$ Clin Microbiol 2001, 39:3705-3708.

20. Niemann S, Harmsen D, Rüsch-Gerdes S, Richter E: Differentiation of clinical Mycobacterium tuberculosis complex isolates by gyrB DNA sequence polymorphism analysis. J Clin Microbiol 2000, 38:3231-3234. 
21. Huard RC, Chitale S, Leung M, Lazzarini LC, Zhu H, Shashkina E, Laal S, Conde MB, Kritski AL, Belisle JT, Kreiswirth BN, Lapa e Silva JR, Ho JL: The Mycobacterium tuberculosis complex-restricted gene cfp32 encodes an expressed protein that is detectable in tuberculosis patients and is positively correlated with pulmonary interleukin-10. Infect Immun 2003, 71:6871-6883.

22. Kim BJ, Lee SH, Lyu MA, Kim SJ, Bai GH, Chae GT, Kim EC, Cha CY, Kook YH: Identification of mycobacterial species by comparative sequence analysis of the RNA polymerase gene (rpoB). J Clin Microbiol 1999, 37:1714-1720

23. Das S, Das SC, Verma R: Occurrence of RD9 region and 500 bp fragment among clinical isolates of Mycobacterium tuberculosis and Mycobacterium bovis. Microbiol Immunol 2007, 51:231-234.

24. Yee D, Valiquette C, Pelletier M, Parisien I, Rocher I, Menzies D: Incidence of serious side effects from first-line antituberculosis drugs among patients treated for active tuberculosis. Am J Respir Crit Care Med 2003, 167:1472-1477.

25. Warren RM, Gey van Pittius NC, Barnard M, Hesseling A, Engelke E, de Kock M, Gutierrez MC, Chege GK, Victor TC, Hoal EG, van Helden PD:

Differentiation of Mycobacterium tuberculosis complex by PCR amplification of genomic regions of difference. Int J Tuberc Lung Dis 2006, 10:818-822

26. Pinsky BA, Banaei N: Multiplex real-time PCR assay for rapid identification of Mycobacterium tuberculosis complex members to the species level. J Clin Microbiol 2008, 46:2241-2246.

27. Rezai MS, Khotaei G, Mamishi S, Kheirkhah M, Parvaneh N: Disseminated Bacillus Calmette-Guerin infection after BCG vaccination. J Trop Pediatr 2008, 54:413-416

28. Kamerbeek J, Schouls L, Kolk A, van Agterveld M, van Soolingen D, Kuijper S, Bunschoten A, Molhuizen H, Shaw R, Goyal M, van Embden J: Simultaneous detection and strain differentiation of Mycobacterium tuberculosis for diagnosis and epidemiology. J Clin Microbiol 1997 35:907-914.

\section{Pre-publication history}

The pre-publication history for this paper can be accessed here: http://www.biomedcentral.com/1471-2334/10/118/prepub

doi: 10.1186/1471-2334-10-118

Cite this article as: Nakajima et al., Identification of Mycobacterium tuberculosis clinical isolates in Bangladesh by a species distinguishable multiplex PCR BMC Infectious Diseases 2010, 10:118

Submit your next manuscript to BioMed Centra and take full advantage of:

- Convenient online submission

- Thorough peer review

- No space constraints or color figure charges

- Immediate publication on acceptance

- Inclusion in PubMed, CAS, Scopus and Google Scholar

- Research which is freely available for redistribution

Submit your manuscript at www.biomedcentral.com/submit
C Biomed Central 